\title{
Newly Constructed Real Time ECG Monitoring System Using LabView
}

\author{
Dr. V. Nandagopal ${ }^{1}$, Dr. V. Maheswari' ${ }^{1}$, C. Kannan ${ }^{2}$ \\ ${ }^{1}$ Department of Electrical and Electronics Engineering, Sreenivasa Institute of Technology and Management Studies, \\ Murukambattu Chittoor, India \\ ${ }^{2}$ Department of Electrical and Electronics Engineering, Arunai Engineering College, Tiruvannamalai, India \\ Email:nandhu050577@gmail.com,magivimal@gmail.com,kannanc305@gmail.com
}

How to cite this paper: Nandagopal, Dr.V., Maheswari, Dr.V. and Kannan, C. (2016) Newly Constructed Real Time ECG Monitoring System Using LabView. Circuits and Systems, 7, 4227-4235. http://dx.doi.org/10.4236/cs.2016.713347

Received: May 6, 2016

Accepted: May 20, 2016

Published: November 29, 2016

Copyright $\odot 2016$ by authors and Scientific Research Publishing Inc. This work is licensed under the Creative Commons Attribution International License (CC BY 4.0).

http://creativecommons.org/licenses/by/4.0/

\begin{abstract}
This paper deals with the removal of noise and base wander in the transfer of ECG data from the patients to the doctor. The process of the project is receiving ECG signals from the patient and reading the data in PC using an Arduino (an open-source electronics prototyping platform based on flexible, easy-to-use hardware and software) board, and then the signal is subjected to the removal of noise and base wander by amplification circuits in LabView (a system design software) software. Thus obtained ECG signal is sent to the doctors using an Ethernet cable or LAN connection. This enables the doctors to monitor any number of patients more accurately by sitting in a single room.
\end{abstract}

\section{Keywords}

Electrocardiography, Arduino, LabView, Noise Removal

\section{Introduction}

ECG (electrocardiogram) is a test which measures the function of a heart. The heart is a muscular organ beats in rhythm to pump the blood throughout the body. Heart's muscle fibers make signals from the sinoatrial node, which is the natural pacemaker of the heart. In an ECG test, heart beating is recorded as electrical impulses are usually shown as waves in the display. This is called ECG test. The recorded waves are the rhythmical function of heart. An embedded wireless health monitoring system is proposed which is made up of wearable monitoring nodes with BLE, a multi-thread mechanism and object oriented method of LabView is proposed [1]. It's proposed a Wireless ECG portable system for remote monitoring for cardiac activity using zigbee technology [2]. Proposed a low cost ECG monitoring system is designed with LabView and FPGA. Bug is 
eliminated in VHDL coding for better performances [3]. Portable and cost effective data acquisition (DAQ) for clinical application developed for finding cardiovascular diseases detections [4]. A 7-lead ECG monitoring system is enabled with smart phones. With clinical intelligent functions, a health risk algorithm is proposed to detect ECG signal abnormities [5]. A U-Healthcare system is developed using various short range technologies and proprietary protocols with zigbee and Bluetooth. 6LoWPAN based communication platform for next generations [6]. Wireless healthcare network platform based on IEEE802.15.4 standard performance analysis of real time monitoring of ECG signals is validated [7]. Heart rate variability (HRV) analysis in premature neonates, a system to acquire and analyze full frequency spectrum was proposed [8]. It's proposed a highly convenient ECG monitoring system for portable, miniature, battery powered with low power consumption [9]. On account of analyzing the recorded heart beat, the heart diseases are identified. This paper improves the accuracy in recording and analyzing the function of heart, using LabView. Electrocardiography is a transthoracic (across the thorax or chest) interpretation that records the heart beatings over a period of time. The electrodes are attached to the surface of the skin which detects and records the heart beatings to an external device. The noninvasive procedure records the activity of the heart, and is the function of electrocardiogram. An ECG test is used to measure the rate and regularity of heartbeats, the size and position of the chambers, the presence of any damage to the heart, and the effects of drugs or devices used to regulate the heart. Probably the ECG tests are performed on diagnostic or research purposes to human hearts. Eventually the test can be performed on animals usually to diagnose or research heart abnormalities. Real time monitoring plays an important role in biomedical engineering, particularly in ECG, EMG, EEG etc. Personal computers have become a standard platform for the requirement of various measurements, tests, standardizations and performances.

Figure 1 shows an ECG signal which is usually small, approximately $1 \mathrm{mV}$. Thus, it is prone to be corrupted by various noises. Power line interference, electrode contact

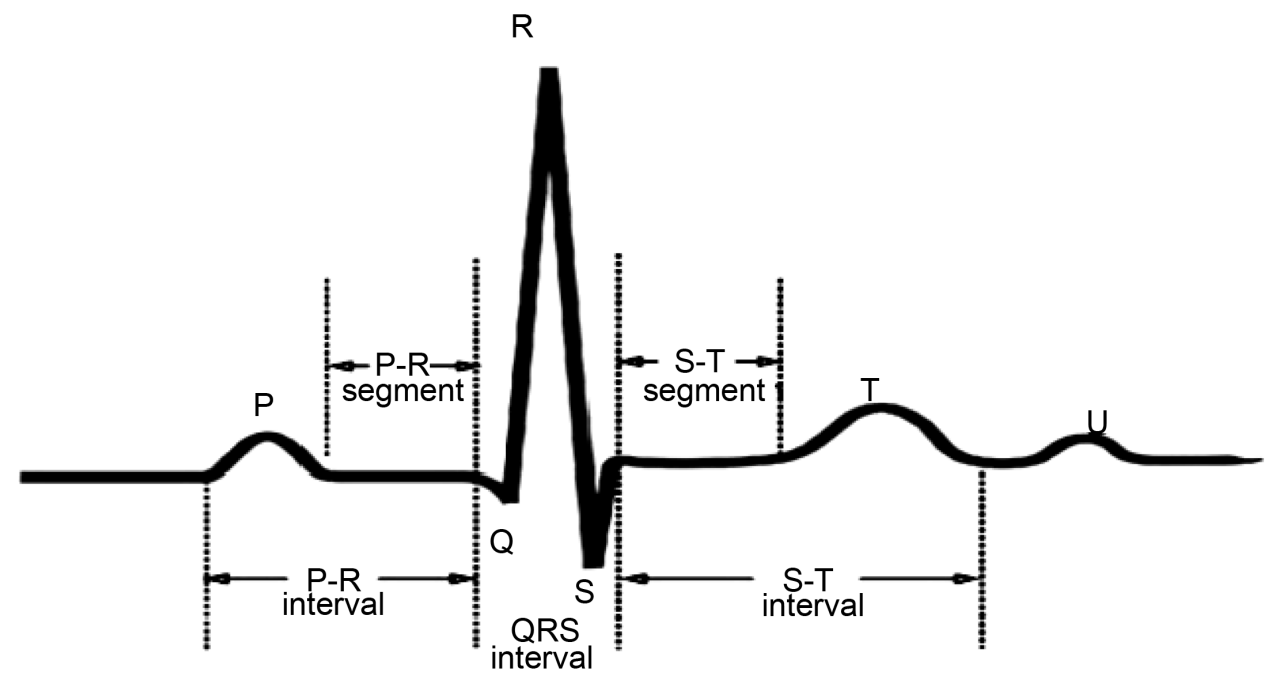

Figure 1. Element of ECG complex. 
noise and motion artifacts are the noise interruptions which probably occur in the ECG signals. Hence, it is necessary to design a good filter system to filter the noises from the ECG signal for attaining better result. Therefore, measuring an ECG signal is not an easy task. Heartbeat is the number of beats per unit of time which is typically expressed as beats per minute (bpm). The body needs to absorb oxygen and excrete carbon dioxide changes either at work or at sleep that the bpm varies man to man. The measurement of heart beat is inevitable to the medical professionals for diagnosing and tracking the condition of patients. The individuals like athletes are interested in monitoring their heart beat for maximum performance during their rigorous training. In Figure 2 electrical activity of heart is shown, heart is the organ which pumps blood throughout the body. It is located in the middle of the thorax, slightly offset to the left and surrounded by the lungs. The heart is composed of four chambers; two atriums and two ventricles. The right atrium receives blood returning to the heart from the whole body. The blood passes through the right ventricle and is pumped to the lungs where it is oxygenated and goes back to the heart through the left atrium, and then the blood passes through the left ventricle and is pumped again for distribution to the entire body through the arteries.

This is a list of events that occur in the heart on each heart beat.

Atrium begins to depolarize.

Atrium depolarizes.

Ventricles begin to depolarize at apex. Atrium re polarizes.

Ventricles depolarize.

$>$ Ventricles begin to re polarize at apex.

Ventricles re polarize.

\section{Software Description}

LabView is system design software (Figure 3 logo) with the tools, enabling engineers and scientists to create, deploy measurement and control the systems. The biomedical toolkit of LabView provides way (express via) for simulation of electrocardiogram (ECG), electromyogram (EMG), electroencephalogram (EEG) and noninvasive blood pressure (NIBP) signal.

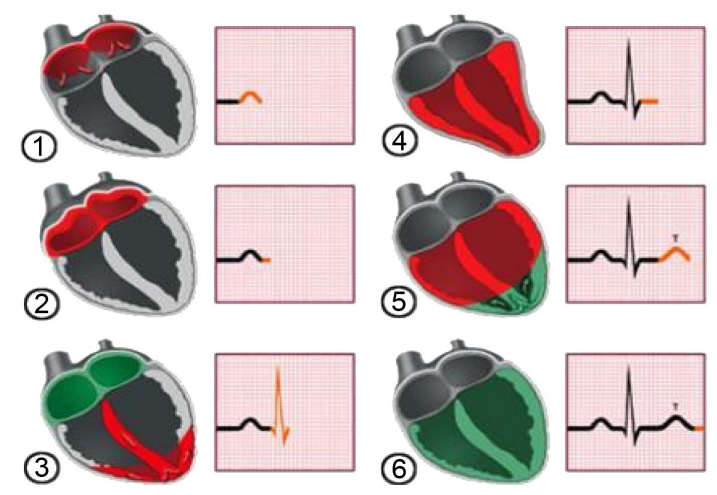

Figure 2. Electrical activity of the heart. 


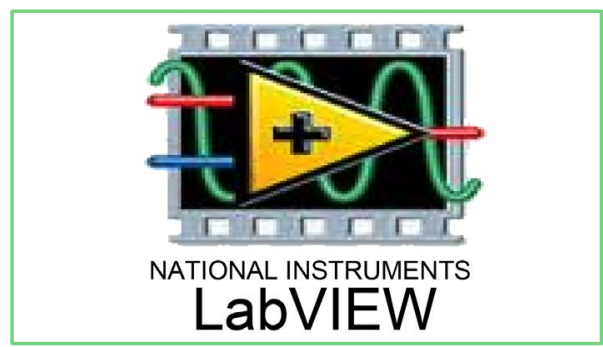

Figure 3. LabVIEW.

The use of LabView and data acquisition in biomedical paves way to the real time monitors systems. It has high performance, low cost of development, more reliability and flexibility. LabView is general purpose software for virtual instrumentation in which other products like dasylab, genie, and alligent vee are followed. In LabView the maintenance and reconfiguration of created instruments are significantly easy and it costs low. While PC based virtual instrumentation is used as a testing platform, it enables recording of real time ECG. Consequently the identification of ECG and transmission of preprocessed data is introduced to a doctor. It is processed through a distributed proposed computation network. This is the PC based proposed monitoring system.

LabView programs are called virtual instruments, or VIs, because their appearance and operation imitate physical instruments, such as oscilloscopes and multi-meters. LabView contains a comprehensive set of tools for acquiring, analyzing, displaying, and storing data and it has tools to help the troubleshoot code which we write. In LabView a user interface or front panel can be built with controls and indicators. Controls are knobs, push buttons, dials, and other input mechanisms. Indicators are graphs, LEDs, and other output displays. After the front panel is built, code using VIs and structures is added to control the front panel objects. The block diagram contains this code.

LabView can be used to communicate with hardware such as data acquisition, vision, and motion control devices, as well as GPIB, PXI, VXI, RS232, and RS485 instruments. The GUI of the system has been developed in Microsoft. NET visual and C++ but it lacks the simultaneous lead illustration of ECG waveform. Its selection and addition of intelligence is for auto diagnose. Integrating dyadic wavelet algorithm in intelligent virtual ECG device, PQRS is detected. Recording and identifying the signals with heart rhythm detection facility and off line analysis of prerecorded ECG signal has been proposed.

In addition to the development of biomedical engineering, the designed system of this paper proposes the automatic removal of noises and filtration of acquired signal on virtual cardiographs. Thus, the system is used for analyzing, identifying and diagnosing the peak QRS automatically.

\section{The Proposed Activity}

The proposed activity in the paper is, picking up an ECG signal (i.e. real time ECG acquisition from a patient), finding R-R intervals, detecting QRS complex peak, reducing 
noise and finding the heart rate. The heart rate is sent to the doctor, using UDP protocol (i.e. server to client transformation). Using the Heart rate, if there is any disease occurrence like sinus arrthymia, Bradycardia, Tachycardia etc., is diagnosed. Thus, the cardiac diseases are diagnosed exclusively and subjected to required treatment.

\section{Methodology}

At Server Using simulator, the ECG signal is picked up and the signal is simulated and shown in Figure 4. Following it, the wavelet analysis is done using Discrete Wavelet Transform (DWT). DWT is any wavelet transform for which the wavelets are discretely sampled. Like other wavelet transforms, this DWT too can be used in Fourier transforms. DWT captures both frequency and location information (location in time). The discrete wavelet transform has a huge number of applications in science, engineering, mathematics and computer science. Significantly, it is used for signal coding that it is to represent a discrete signal in a more redundant form, often as a preconditioning for data compression. In signal processing, Practical applications also can be found such as; accelerations for gait analysis, digital communications etc. After wavelet is analyzed, signals are merged, processed, denoised, and detrended. Then, R-R interval peak is detected, heart rate is calculated and server-client information is transferred. LabView Biomedical Toolkit in PC provides an ECG Feature Extractor VI, and an ECG Feature Extractor application for users to extract ECG features conveniently (Add link for "How to use ECG Feature Extractor" here). Using the toolkit, either QRS can be detected only or all supported ECG features, including R position, R amplitude, is level, QRS onset, QRS offset, P onset, P offset, T onset and T offset can be detected is shown in Figure 4.

\section{Interfacing of Hardware with LabView}

The ECG Feature Extractor detects all beats (R waves) in the signal, and extracts other features for every beat. The accuracy of detecting $\mathrm{R}$ waves is the paramount requirement,

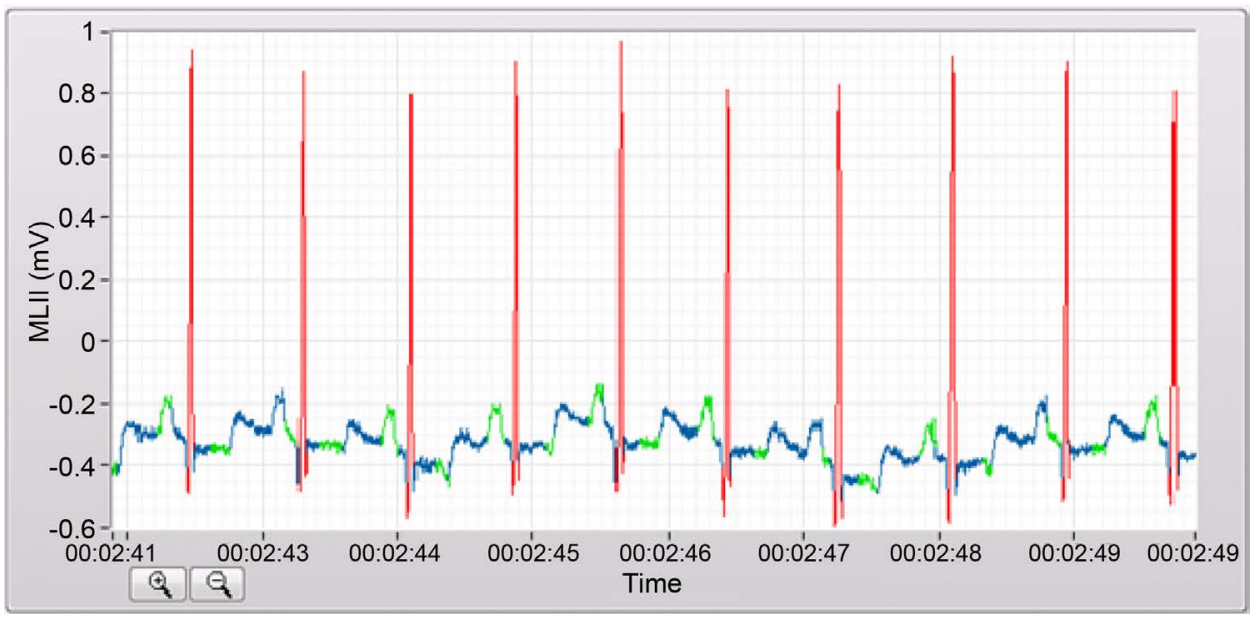

Figure 4. ECG multi resolution analysis and implementation of QRS detection. 
is established. Normal ECG signals can be easily detected, while abnormal morphology makes the detection difficult. Abnormal morphology occurs for the patient who has some specific heart diseases. Thus, some signal enhancement (preprocessing) is needed before feature extraction. The preprocessed ECG signal is used to detect position of $\mathrm{R}$ waves. All other features will be extracted then using original signal, because the signal enhancement may change these features. Figure 5 shows the output of ECG waveform in lab view.

The special features of proposed work able to acquire the signal from the ECG device. Display of continuous time ECG signal traces Able to filter the signal acquired through different types of filter. The ECG output from the circuit will be the input data of DAQ Assistant in the LabView where the data can be stored or display in a graph. The ECG signal will be multiplied with desired gain and band stop filter used to remove frequency in between $50 \mathrm{~Hz}$ to $60 \mathrm{~Hz}$ [4]. Figure 6 shows the Complete ECG system hardware setup.

The analog signal of ECG will be converted into digital signal by ADC and stored in personal computers. We have proposed graphical user interface in LabView software to display ECG wave forms to display and record over a period of time. Figure 7 shows the ECG waveform in LabView software.

Figure 8 shows the block diagram of ECG system using system on chip.

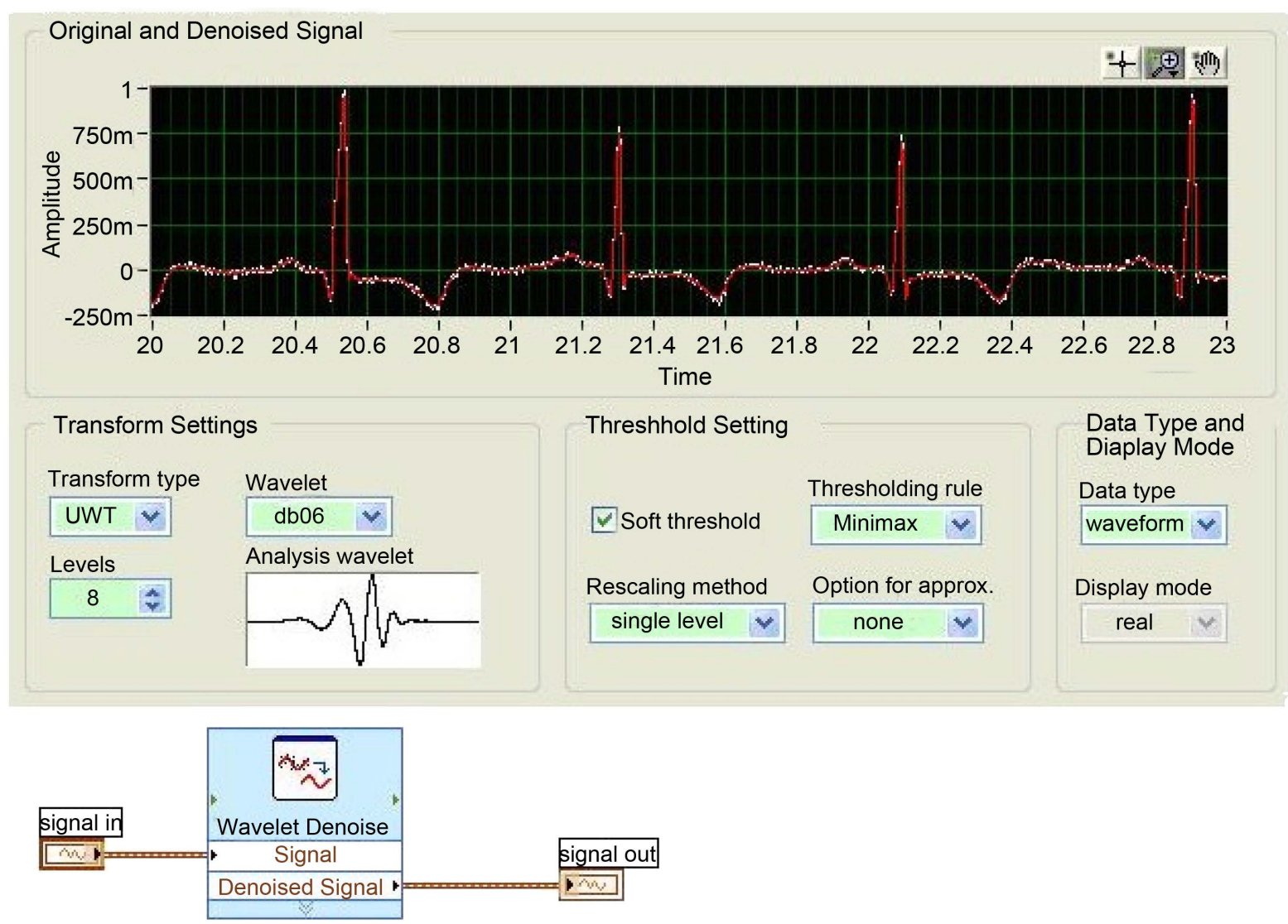

Figure 5. Output of ECG wave in LabView software. 


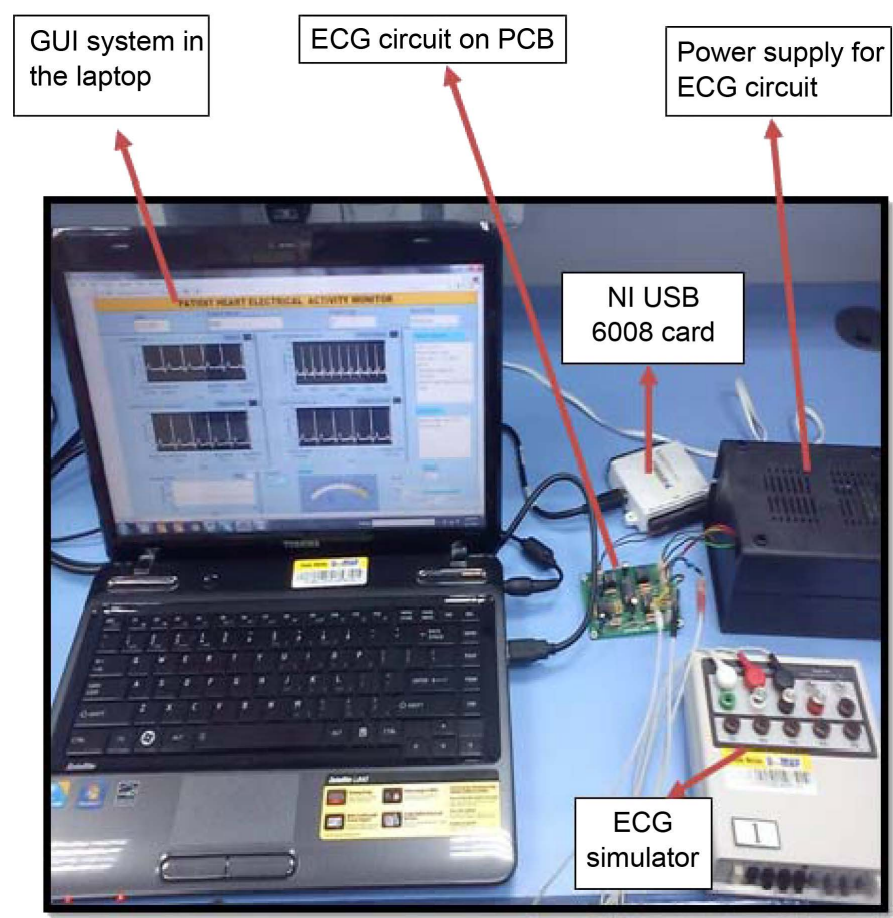

Figure 6. Complete ECG system using proposed design.

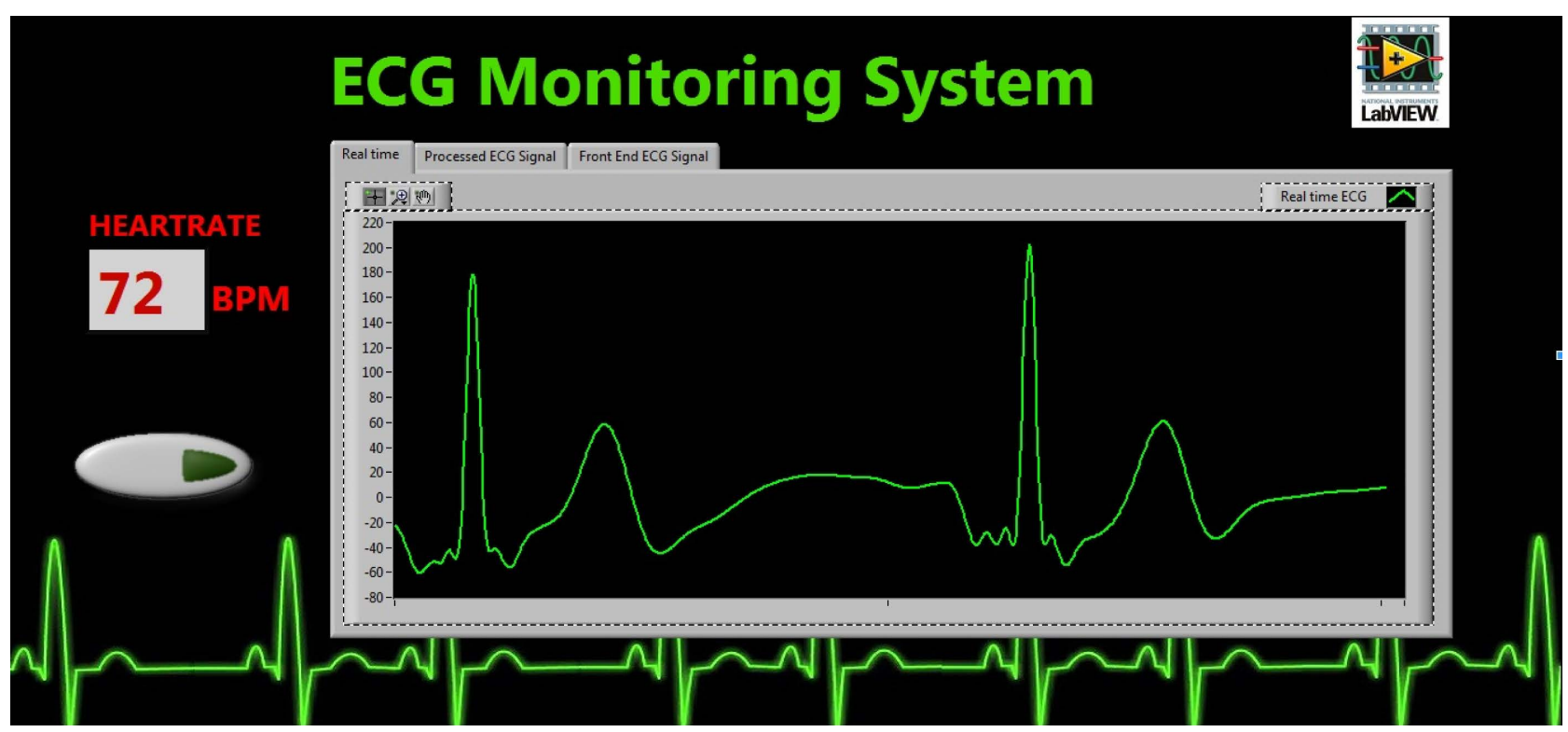

Figure 7. ECG acquisition using LabView software.

\section{Conclusion}

In this paper ECG monitoring system proceeds a system for reducing noise and base wander in ECG data. An ECG signal is taken up from the patient and using an Arduino (an open-source electronics prototyping platform based on flexible, easy-to-use hardware and software) board the data are read in PC. In LabView, with the access of amplification circuit's system design software, the noise and base wander are removed. 


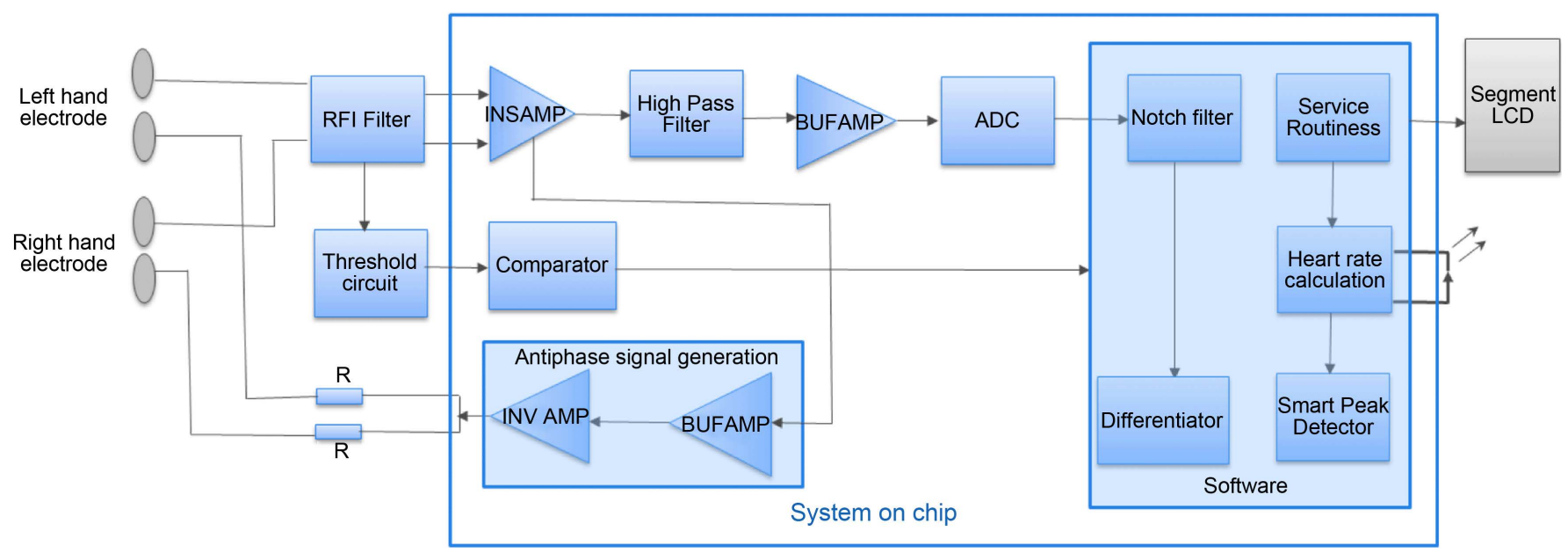

Figure 8. ECG system using system on chip.

Now the obtained ECG signal is sent from the patients recording system to doctor using an Ethernet cable or LAN connection. Consequently, the doctor can assess multiple number of ECG patients at an instant moment.

\section{References}

[1] Chen, X.X. Xie, J.B., Fang, Z. and Xia, S.H. (2015) Low Power Electrocardiography and Impedance Cardiography Detection System Based on LabView and Bluetooth Low Energy. IEEE 2015 IET International Conference on Biomedical Image and Signal Processing (ICBISP 2015), Beijing, 19 November 2015, 1-4. https://doi.org/10.1049/cp.2015.0798

[2] Priya, L., Hariprasad, R. and Raghul, R. (2014) Real Time Monitoring of Vital Signs Using Wireless Technique. IEEE Green Computing Communication and Electrical Engineering, 6-8 March 2014, 1-7.

[3] Chabchoub, S., Mansouri, S. and Salah, R.B. (2015) Biomedical Monitoring System Using LabVIEW FPGA. IEEE Information Technology and Computer Applications Congress, 11-13 June 2015, 1-5.

[4] Murugappa, M., Thirumani, R., Omar, M.I. and Murugappan, S. (2014) Development of Cost Effective ECG Data Acquisition System for Clinical Applications Using LabView. IEEE Signal Processing \& Its Applications (CSPA), 100-105.

[5] Ga, H.Q., Duan, X.H., Guo, X.Q. and Huang, A.P. (2013) Design and Tests of a Smartphones-Based Multi-Lead ECG Monitoring System. IEEE 2013 35th Annual International Conference of the IEEE Engineering in Medicine and Biology Society (EMBC), 267-2270.

[6] Touati, F., Tabish, R. and Mnaouer, A.B. (2013) Towards u-Health: An Indoor 6LoWPAN Based Platform for Real-Time Healthcare Monitoring. 2013 6th Joint IFIP IEEE Wireless and Mobile Networking Conference (WMNC), 1-4.

[7] Lin, W. (2011) Real Time Monitoring of Electrocardiogram through IEEE802.15.4 Network. IEEE 2011 8th International Conference \& Expo on Emerging Technologies for a Smarter World (CEWIT), 1-6. https://doi.org/10.1109/CEWIT.2011.6135891

[8] Oliu, M.S.C., Bello, E. and Bohórquez, J. (2013) Development of a System for the Assessment of Heart Rate Variability in the NICU. IEEE 2013 29th Southern Biomedical Engineering Conference (SBEC), 25-26.

[9] Jeong, D.-U. (2008) Development of a Technique for Cancelling Motion Artifact in Ambu- 
latory ECG Monitoring System. IEEE Convergence and Hybrid Information Technology, 954-961.

\section{Submit or recommend next manuscript to SCIRP and we will provide best service} for you:

Accepting pre-submission inquiries through Email, Facebook, LinkedIn, Twitter, etc. A wide selection of journals (inclusive of 9 subjects, more than 200 journals)

Providing 24-hour high-quality service

User-friendly online submission system

Fair and swift peer-review system

Efficient typesetting and proofreading procedure

Display of the result of downloads and visits, as well as the number of cited articles

Maximum dissemination of your research work

Submit your manuscript at: http://papersubmission.scirp.org/

Or contact cs@scirp.org 